EXTENDED REPORT

\title{
Interpreting the multifocal visual evoked potential: the effects of refractive errors, cataracts, and fixation errors
}

\author{
B J Winn, E Shin, J G Odel, V C Greenstein, D C Hood
}

Br J Ophthalmol 2005;89:340-344. doi: 10.1136/bjo.2004.047910

See end of article for authors' affiliations

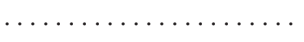

Correspondence to:

D C Hood, Department of Psychology, 405

Schermerhorn, Columbia

University, New York, NY

10027, USA; dch3@

columbia.edu

Accepted for publication

1 August 2004 Aim: To understand how refractive errors, cataracts, and fixation errors affect multifocal visual evoked
potential (mfVEP) responses.
Methods: Monocular mfVEP responses were obtained using a pattern reversal dartboard display. For the Methods: Monocular mfVEP responses were obtained using a pattern reversal dartboard display. For the
control condition, visual acuity was corrected to $\geqslant 20 / 20$ and foveal fixation was maintained. The right eye was tested under the following conditions: simulated refractive error, simulated cataract, steady eccentric fixation, and unsteady fixation.

Results: No subject demonstrated significant abnormalities under control conditions. For the simulated refractive error condition, significant centrally located abnormalities were seen for all subjects. For the simulated cataract condition, significant abnormalities were found for three subjects. The steady eccentric fixation condition yielded abnormalities in both eyes for all subjects while the unsteady fixation condition yielded significant central abnormalities in the tested eye. With eccentric and unsteady fixation conditions, all subjects had at least one sector with a waveform polarity reversal.

Conclusions: While the mfVEP is a useful tool for identifying local optic nerve damage or ruling out nonorganic aetiology of visual field defects, factors such as uncorrected refractive errors, cataract, eccentric fixation, and unsteady fixation can produce apparent field defects on the mfVEP. With care, these problems can be correctly identified.
$\mathrm{T}$ he multifocal visual evoked potential (mfVEP), based upon Sutter's multifocal electroretinogram technique, was developed by Baseler and colleagues ${ }^{2}$ to provide a method for obtaining local visual evoked potential (VEP) responses from the visual field. The technique combines conventional VEP recording techniques with a display that is subdivided into a number of regions. Each of these regions is an independent stimulus controlled by specialised software. From a single, continuous EEG signal, a mathematical algorithm extracts the VEP response generated by each region.

Baseler $\mathrm{et} \mathrm{al}^{2}$ suggested that the clinical utility of the MfVEP was limited because of the variation of responses obtained from identical locations in normal individuals. However, the test was improved by Graham et $a l^{3}$ and Hood et $a l^{45}$ who introduced an interocular comparison technique based on the finding that mfVEPs elicited from the right and left eyes of individuals with no abnormalities of the visual system are virtually identical. More recently, it has been demonstrated that a strictly monocular test, if properly analysed, could also have clinical utility. ${ }^{6}{ }^{7}$ The mfVEP is becoming recognised as a useful tool for diagnosing and following optic nerve/ganglion cell damage (glaucoma, optic neuritis, optic neuropathies) and for ruling out non-organic aetiology of visual field defects. ${ }^{3-11}$

In general, the mfVEP has been conducted on subjects with good visual acuity, no significant lenticular opacities, and steady central fixation. It is well documented that two different forms of blur-uncorrected refractive error ${ }^{12}{ }^{13}$ and cataract $^{14}{ }^{15}$-and fixation errors ${ }^{16}$ can yield erroneous results with static automated perimetry. Blurred vision also has been shown to decrease the amplitude of the conventional pattern reversal VEP. ${ }^{17}$ As the mfVEP is becoming used more widely, it is important to understand how uncorrected refractive errors, cataracts, and fixation errors can affect the results. The purpose of this paper is to understand how these conditions may affect the mfVEP.

\section{METHODS}

Subjects

Eight individuals with $\geqslant 20 / 20$ corrected visual acuity and with no known abnormalities of the visual system participated in the study. They ranged in age from 20 to 60 years (mean 33 (SD 14) years). Procedures adhered to the tenets of the Declaration of Helsinki and the protocol was approved by the institutional board of research associates of Columbia University.

\section{Display}

Figure $1 \mathrm{~A}$ is a schematic of the scaled, dartboard display, $44.5^{\circ}$ of visual angle in diameter. The dartboard, a standard option produced by Veris software from EDI (ElectroDiagnostic Imaging, San Mateo, CA, USA), contained 60 sectors, each with 16 checks, eight white $\left(200 \mathrm{~cd} / \mathrm{m}^{2}\right)$ and eight black $\left(<1 \mathrm{~cd} / \mathrm{m}^{2}\right)$. A cross subtending $2^{\circ}$ served as the fixation target for all conditions except for the unsteady fixation condition in which a central circle with a $3^{\circ}$ radius served as the fixation guide.

\section{Recording}

Three channels of recording were obtained using gold cup electrodes. Recording electrodes were placed at the inion, $4 \mathrm{~cm}$ above the inion, and at two lateral locations up $1 \mathrm{~cm}$ and over $4 \mathrm{~cm}$ from the inion. By subtracting different combinations of pairs of channels, three additional "derived" channels were obtained. For details see Hood et al. ${ }^{8}{ }^{18}$

The VEP was recorded with cut offs set at $3 \mathrm{~Hz}$ and $100 \mathrm{~Hz}$ (1/2 amplitude; Grass preamplifier P511J, Quincy, MA, USA). For a given condition, two 7 minute recordings were obtained for monocular stimulation of each eye. For each eye, the two recordings were averaged and the mfVEP responses extracted with the Veris 4.x software from EDI (San Mateo, CA, USA).

Abbreviations: $m f V E P$, multifocal visual evoked potential; RMS, root mean square; SNR, signal to noise ratio; VEP, visual evoked potential 


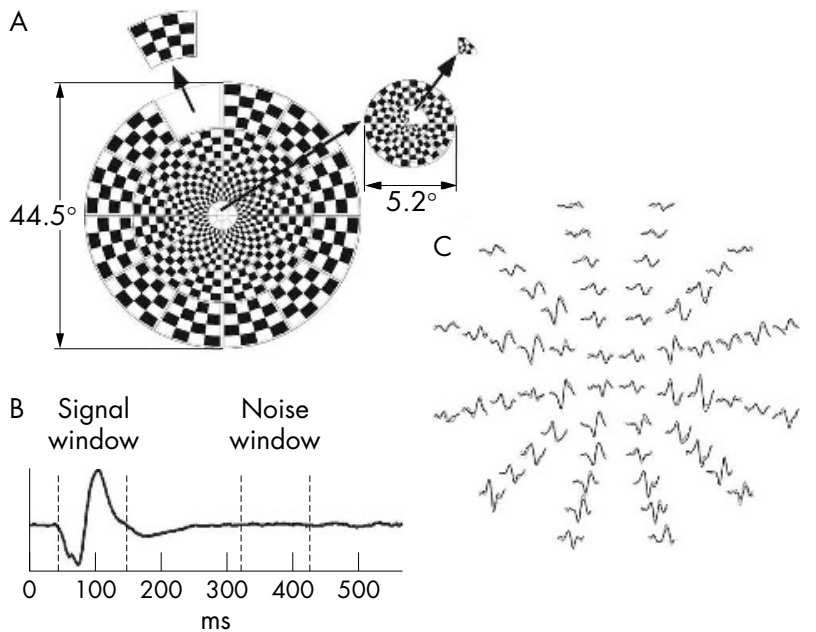

Figure 1 (A) Each of the 60 sectors of the display is an independent stimulus with 16 checks, eight black and eight white. (B) An example of a $\mathrm{mfVEP}$ record from $0 \mathrm{~ms}$ to $500 \mathrm{~ms}$. Signal and noise windows are demarcated by vertical broken lines. (C) The mfVEP responses from an individual with normal vision and steady central fixation are shown for monocular stimulation of the right (thick grey) and left (thin black) eye.

In addition, the mfVEPs were low pass filtered (sharp cut off at $35 \mathrm{~Hz}$ ) and analysed offline with programs written in MATLAB (Mathworks Inc, MA, USA).

\section{Conditions}

For each subject, mfVEPs were recorded under the following conditions: control, simulated refractive error, simulated cataract, steady eccentric fixation, and unsteady fixation. For each condition, the left eye served as a control $(\geqslant 20 / 20$ visual acuity and steady, central fixation) while the right eye served as the test eye. The mfVEP system has an adjustable lens $(-5$ to +5 dioptres) through which each subject views the stimulus. For the control condition, each subject was instructed to adjust the lens such that the stimulus was as clear as possible and to fixate on the central target. Acuity was checked with a scaled Snellen chart placed on the screen and was $\geqslant 20 / 20$ for all subjects. For the refractive error condition, the same Snellen chart was used while each subject adjusted the focus in the convex $(+)$ direction away from his $\geqslant 20 / 20$ setting such that the acuity was $20 / 50$. This setting was then used for the simulated refractive error condition. For all other conditions each subject viewed the stimulus through his setting $(\geqslant 20 / 20)$ of the focusing lens. To simulate a cataract, the display was viewed through a 0.4 Bangerter occlusion foil placed $3 \mathrm{~cm}$ in front of the right eye. To simulate steady eccentric fixation, the subject was instructed to fixate on a target $3^{\circ}$ inferonasal to the central fixation target. To simulate unsteady fixation, the subject was instructed to rotate fixation with the right eye around the perimeter of a centrally located circle with a $3^{\circ}$ radius.

\section{Analysis}

Interocular probability plots

Root mean square (RMS) amplitudes were calculated for each mfVEP response over a time interval from $45 \mathrm{~ms}$ to $150 \mathrm{~ms}$ (fig 1B). To determine if responses were significantly smaller in the right eye compared to the left eye, the ratio of the RMS amplitude from stimulation of the right eye to the RMS amplitude from stimulation of the left eye for each stimulus sector was calculated (that is, $\left[\mathrm{RMS}_{\mathrm{RE}} / \mathrm{RMS}_{\mathrm{LE}}\right]$ ). The interocular RMS ratio (RE/LE ratio) for each location was then compared to the RE/LE ratio values from 30 control subjects run under standard conditions using a Z-score
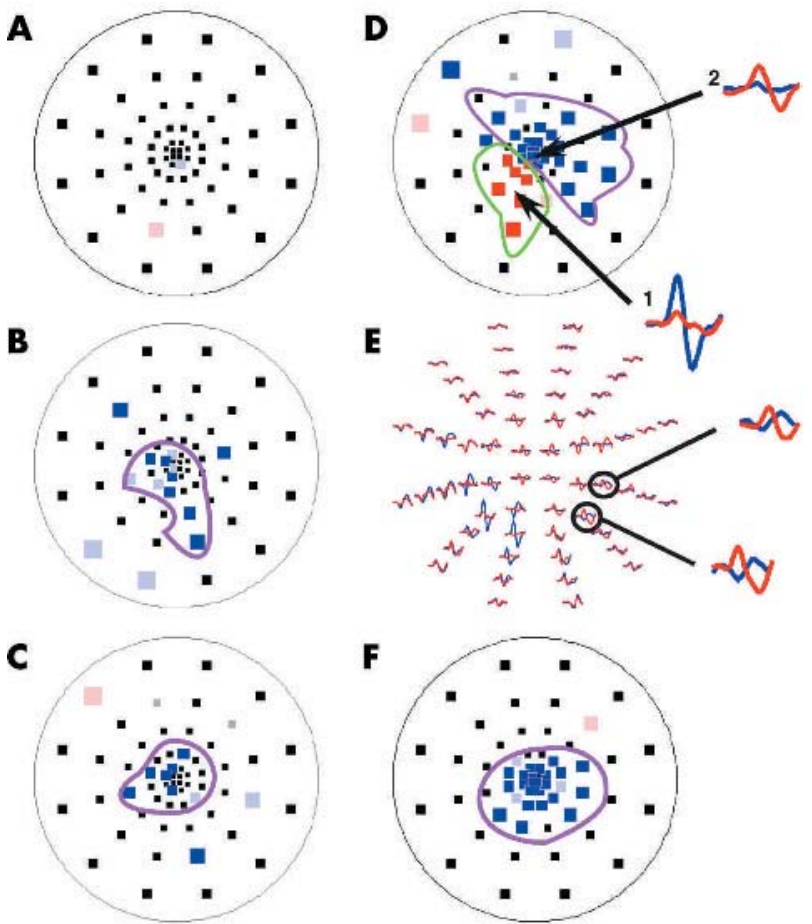

Figure 2 (A) The interocular probability plot for a subject with normal vision under control conditions. Each square shows the centre of one of the $60 \mathrm{mfVEP}$ sectors. Blue indicates that the response of the right eye was significantly smaller than from the left while red indicates that the response from the left eye was significantly smaller than from the right. Desaturated colours indicate responses outside $5 \%$ cut offs while saturated colours indicate responses outside 1\% cut offs. (B) The interocular probability plot for a subject with a refractive error of approximately $20 / 50$. The area of abnormality is circumscribed in magenta. (C) The interocular probability plot for a subject under simulated cataract conditions. The area of abnormality is circumscribed in magenta. (D) The interocular probability plot for a subject under steady eccentric fixation conditions. The area of apparent abnormality in the left eye is circumscribed in green while the area of apparent abnormality in the right eye is circumscribed in magenta. Examples of the relative amplitudes of the mfVEP responses in these areas are shown in insets 1 and 2. For this figure and all other figures red traces indicate waveforms from stimulation of the left eye while blue traces indicate waveforms from the right. (E) The interocular waveform trace array for a subject under steady eccentric fixation conditions. Examples of interocular waveform polarity reversals are shown in the insets. (F) The interocular probability plot for a subject under unsteady, central fixation conditions. The apparent abnormality is circumscribed in magenta.

analysis and an interocular probability plot was derived. All control subjects had $\geqslant 20 / 20$ visual acuity and no known abnormalities of the visual system. Their ages ranged from 20 to 62 years with mean age 36 (SD 13). Figure $2 \mathrm{~A}$ is an example of an interocular probability plot for a normal subject under control conditions. (The responses from both eyes of this subject are shown in fig 1C.) In these plots, each check represents the centre of one of the stimulus sectors of the display. Black squares indicate areas where the RE/LE ratio is within normal limits. Red squares indicate areas where the mfVEP amplitudes from stimulation of the left eye are significantly smaller than from the right. Blue squares indicate areas where amplitudes from stimulation of the right eye are significantly smaller than from the left. A desaturated colour indicates that the mfVEP amplitude at that location was significantly smaller at the $5 \%$ level while a saturated colour indicates it was smaller at the $1 \%$ level. For more details, see Hood et al. ${ }^{58}$ 
Table 1 Summary of cluster analysis (interocular test/monocular test)

\begin{tabular}{|c|c|c|c|c|c|c|c|c|}
\hline & \multicolumn{8}{|c|}{ Subject } \\
\hline & 1 & 2 & 3 & 4 & 5 & 6 & 7 & 8 \\
\hline Control & $\mathrm{N} / \mathrm{N}$ & $\mathrm{N} / \mathrm{N}$ & $\mathrm{N} / \mathrm{N}$ & $\mathrm{N} / \mathrm{N}$ & $\mathrm{N} / \mathrm{N}$ & $\mathrm{N} / \mathrm{N}$ & $\mathrm{N} / \mathrm{N}$ & $\mathrm{N} / \mathrm{N}$ \\
\hline Refractive error & $\mathrm{A} / \mathrm{N}$ & $\mathrm{A} / \mathrm{N}$ & $\mathrm{A} / \mathrm{N}$ & $\mathrm{A} / \mathrm{N}$ & $\mathrm{A} / \mathrm{N}$ & $\mathrm{A} / \mathrm{A}$ & $\mathrm{A} / \mathrm{A}$ & $\mathrm{A} / \mathrm{A}$ \\
\hline Simulated cataract & $\mathrm{N} / \mathrm{N}$ & $\mathrm{A} / \mathrm{N}$ & $\mathrm{A} / \mathrm{N}$ & $\mathrm{N} / \mathrm{N}$ & $\mathrm{N} / \mathrm{N}$ & $\mathrm{A} / \mathrm{A}$ & $\mathrm{N} / \mathrm{A}$ & $\mathrm{N} / \mathrm{N}$ \\
\hline Steady eccentric fixation & $\mathrm{A} / \mathrm{A}$ & $\mathrm{A} / \mathrm{N}$ & $\mathrm{A} / \mathrm{A}$ & $\mathrm{A} / \mathrm{A}$ & $\mathrm{A} / \mathrm{N}$ & $\mathrm{A} / \mathrm{A}$ & $\mathrm{A} / \mathrm{A}$ & $\mathrm{A} / \mathrm{A}$ \\
\hline Unsteady central fixation & $\mathrm{A} / \mathrm{A}$ & $\mathrm{A} / \mathrm{A}$ & $\mathrm{A} / \mathrm{A}$ & $\mathrm{A} / \mathrm{N}$ & $\mathrm{A} / \mathrm{N}$ & $\mathrm{A} / \mathrm{A}$ & $\mathrm{A} / \mathrm{A}$ & $\mathrm{A} / \mathrm{A}$ \\
\hline Mean SNR & 4.70 & 4.59 & 4.65 & 6.00 & 6.12 & 2.78 & 2.61 & 3.28 \\
\hline
\end{tabular}

Monocular probability plots

A signal to noise ratio (SNR) was calculated for each of the 60 stimulus sector positions for each eye. ${ }^{8}{ }^{19}$ Briefly, to obtain the SNR for an individual response, the RMS amplitude of each response, described above, was divided by a measure of the amplitude of the noise. The noise measure was obtained, for each eye of each individual, as the mean of the 60 RMS amplitudes of the records from $325 \mathrm{~ms}$ to $430 \mathrm{~ms}$, a region of the record virtually without any mfVEP response (fig 1B). The 60 SNRs for each eye (one SNR corresponding to each of the sectors of the stimulus) were then compared with the SNRs of 30 normal subjects at each of these positions using a Z-score analysis. Probability plots were then generated for each eye. Black squares indicate areas where the SNRs are within normal limits. For the left eye monocular probability plots, a red square indicates an area where the SNR is significantly smaller than the average SNR from the group of normals at that sector. Blue squares are used in the same manner for the right eye plots. Desaturated colours represent areas significant outside a 5\% limit while saturated colours represent areas significant outside $1 \%$. Since the monocular test is based on SNR, it is expected that abnormalities will be detected more frequently in subjects with inherently small mean SNRs; likewise, fewer abnormalities will be detected in subjects with inherently large mean SNRs. For more details, see Hood et al. ${ }^{8}$

\section{Cluster analysis}

To increase specificity with automated visual field testing, it is common to define a "cluster" of points as abnormal if they collectively meet some criteria. ${ }^{20}{ }^{21}$ The specificities of both the monocular and interocular tests are also improved by employing a cluster criterion to define an abnormal test. ${ }^{6} 722$ For both the interocular and the monocular probability plots, a region was classified as abnormal if three or more contiguous points were significant outside $5 \%$, with at least one outside $1 \% .^{6723}$

\section{Interocular waveform analysis}

For every test condition, the mfVEP response waveform from stimulation of the right eye was compared to that of the left eye at each of the 60 stimulus sector positions. Sectors where the waveform polarity from stimulation of the left eye was opposite to that of the right were considered abnormal. Although these waveform reversals can be detected by visual inspection, we used a cross correlation technique. To avoid artefacts secondary to noise (such as alpha noise with waveforms out of phase with true mfVEP waves) only those pairs with a SNR $\geqslant 1.5$ and a cross correlation index between 0 and -1 were categorised as having interocular polarity reversals. Waveform analyses performed on the trace array for the derived "horizontal" channel (that is, the difference between the two laterally placed electrodes) were found to be the most selective for these interocular polarity reversals.

\section{The best array}

All analyses (other than the waveform analysis performed on the horizontal channel) were performed on the "best" of the responses from the six channels as previously described. ${ }^{818}$

\section{RESULTS}

\section{Control condition}

None of the eight subjects demonstrated any significant abnormalities (clusters) on interocular or monocular probability plots (for example, fig 2A). In addition, none of the subjects demonstrated interocular waveform polarity reversals.

\section{Simulated refractive error condition}

Table 1 summarises the abnormalities by condition and subject along with the average SNRs for each subject's mfVEP of the right eye under the control condition. The results of the interocular and monocular cluster analyses are indicated for each subject and condition with " $\mathrm{A}$ " and " $\mathrm{N}$ " denoting abnormal and normal, respectively. For the refractive error condition (second row in table 1), all subjects demonstrated abnormalities on interocular probability plots and these were predominantly centrally located (for example, fig 2B). Only three of the subjects $(6,7$, and 8$)$ demonstrated significant abnormalities on monocular probability plots for the right (experimental) eye and these subjects had the smallest mean SNRs.

Table 2 summarises the results of the interocular waveform analysis. For each subject and condition, the number of

Table 2 Summary of interocular waveform analysis. Shown are the number of traces (of 60) for each subject and condition with interocular waveform reversals

\begin{tabular}{|c|c|c|c|c|c|c|c|c|}
\hline & \multicolumn{8}{|c|}{ Subject } \\
\hline & 1 & 2 & 3 & 4 & 5 & 6 & 7 & 8 \\
\hline Control & 0 & 0 & 0 & 0 & 0 & 0 & 0 & 0 \\
\hline Refractive error & 0 & 0 & 0 & 1 & 0 & 0 & 0 & 0 \\
\hline Simulated cataract & 0 & 0 & 0 & 0 & 0 & 0 & 0 & 0 \\
\hline Steady eccentric fixation & 6 & 10 & 9 & 14 & 14 & 6 & 5 & 10 \\
\hline Unsteady fixation & 4 & 2 & 12 & 4 & 7 & 1 & 3 & 3 \\
\hline
\end{tabular}


sectors out of the interocular array of 60 that have polarity reversals is shown. For all subjects and all responses, there was only a single interocular waveform polarity reversal for the refractive error condition.

\section{Simulated cataract condition}

Three subjects $(2,3,6)$ demonstrated significant abnormalities on interocular probability plots (table 1) and these defects were again predominantly centrally located (for example, fig 2C). The two subjects (6 and 7) with the lowest mean SNRs in table 1 demonstrated significant abnormalities on monocular probability plots for the right eye. None of the subjects demonstrated interocular waveform polarity reversals (table 2 ).

\section{Steady eccentric fixation condition}

All subjects demonstrated a particular bilateral abnormality on interocular probability plots. In each case there was an area of apparent decreased amplitude for the left eye where the right eye was fixating eccentrically and an area of apparent decreased amplitude for the right eye in the central sectors and the paracentral sectors opposite the direction of eccentric fixation (for example, fig 2D). Six of the eight subjects (all but subjects 2 and 5) demonstrated significant paracentral abnormalities on monocular probability plots for the right eye (table 1). All subjects demonstrated interocular waveform polarity reversals in five or more sectors (table 2 ). The insets in figure $2 \mathrm{E}$ show examples of polarity reversals.

\section{Unsteady fixation condition}

All subjects demonstrated large areas with central abnormalities in the right eye on interocular probability plots (for example, fig 2F). Six subjects demonstrated significant central abnormalities on monocular probability plots in the right eye (table 1). (Subjects 4 and 5 who had the highest average SNRs did not.) All subjects demonstrated interocular waveform polarity reversals in one or more sectors (table 2 ).

\section{DISCUSSION}

\section{The effect of blur}

For the simulated refractive error condition (dioptric blur), all subjects showed an abnormality in the mfVEP of the eye that was blurred when compared with the other eye (for example, fig 2B). For the simulated cataract condition (diffusive blur), three of the eight subjects demonstrated abnormalities on interocular plots (fig 2C).

Both refractive errors and cataracts blur the stimulus. Whether dioptric or diffusive, blur can be thought of as a spatial frequency filter selectively eliminating high spatial frequencies. The greater the blur, the greater the loss of high spatial frequency resolution. The amplitude of the response in
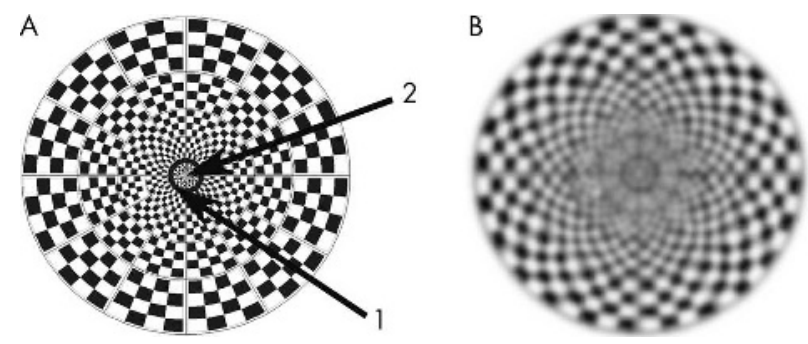

Figure 3 (A) The mfVEP display. Arrow 1 marks the position of fixation of the right eye for the steady, eccentric fixation condition. Arrow 2 marks the position of fixation of the left eye for all conditions. Note the relative difference in size of the stimulus sectors at fixation for the two eyes. (B) A blurred mfVEP display created by a computerised high spatial frequency filter. Note that pattern resolution is virtually lost in the central sectors but relatively preserved in the peripheral sectors. a pattern reversal mfVEP is dependent on the visual system's ability to resolve the pattern in each sector. Owing to the "cortical scaling" of the mfVEP stimulus, the central sectors have higher spatial frequencies relative to the more eccentric sectors. Consequently, removing the higher spatial frequency portion of the stimulus by blurring selectively reduces the ability to resolve the central sectors of the mfVEP. Although created by a computerised high spatial frequency filter rather than a lens or occlusion foil, it is easy to see how the resolution of the central sectors of the stimulus is lost with blur by comparing figure $3 \mathrm{~B}$ with $3 \mathrm{~A}$. Thus, when interpreting a central abnormality on the mfVEP, it is important to rule out cataract and ensure that the subject has been correctly refracted for the viewing distance. In addition, the subject should be instructed to concentrate on resolving the pattern of the central sectors of the display to avoid "defocusing" during the recording.

While the visual acuity for the refractive error condition was $20 / 50$, the acuity with the Bangerter foils was approximately 20/40. Thus, we might expect that the number of abnormalities for the refractive error condition would be close to that found for the cataract condition. In fact, for the refractive error condition, three of the subjects demonstrated an abnormality on monocular probability plots while in the simulated cataract condition, two did. (It is not a coincidence that these subjects also have the smallest average SNRs of the eight . The monocular test is not as sensitive for subjects with high SNRs as the interocular test. ${ }^{7}$ ) However, for the refractive condition all eight subjects showed abnormalities on the interocular plot while only three showed abnormalities for the cataract condition. It is not clear whether this difference reflects the small difference in visual acuity or a fundamental difference in the effects of blur due to cataracts compared to poor correction.

\section{The effect of fixation}

As with the case of blur, much of the effect of eccentric fixation on the mfVEP has to do with the cortical scaling of the stimulus. Each subject was instructed to fixate $3^{\circ}$ inferonasal from the centre in the right eye. In this case, the fovea RE views a relatively large stimulus sector (fig 3A, arrow 1) while the fovea LE views small central sectors (fig 3A, arrow 2). The area inscribed in green in figure 2D represents an apparent abnormality in the left eye. The mfVEPs for the two eyes differ in this region since it was viewed by the fovea RE but by the parafovea LE (fig 2D, inset 1). Likewise, the central sectors of the stimulus (circumscribed in magenta in fig 2D) were viewed by the fovea LE but by the parafovea RE and so here the responses RE will be erroneously decreased in amplitude (fig 2D, inset 2).

To test the effect of unsteady fixation, each subject rotated fixation around a $3^{\circ}$ circle ( shown in thick black, fig $3 \mathrm{~A}$ ) with the right eye while the left eye was kept steadily fixating centrally. In this case central sectors were always viewed by the parafovea RE, but by the fovea LE. By the same logic as above, it can be expected that the relative amplitudes of the central sectors would be abnormally small as they indeed were in the case of the right eye (fig $2 \mathrm{~F}$, circumscribed in magenta). Abnormalities in mfVEP responses secondary to unsteady fixation have previously been reported by Seiple et al and Menz et al. ${ }^{24} 25$

Because signals from the primary visual cortex dominate the mfVEP, ${ }^{26-28}$ the waveform polarities of the responses frequently reverse across the horizontal and vertical meridians of the visual field depending on the orientation of the electrodes. $^{2428} 29$ In our study this phenomenon was most frequently encountered in waveform arrays from the lateral channel. If one eye deviates in fixation from the other, then the waveform polarity in one or more sectors along these 
meridians can differ for the two eyes. In fact, all subjects tested under the steady $3^{\circ}$ eccentric fixation and unsteady fixation conditions demonstrated such abnormalities. (See fig 2E, inset; table 2; and also fig $4 \mathrm{~b}$ in Seiple et al. ${ }^{24}$ ) Of the three conditions requiring steady central fixation, only a single interocular waveform polarity reversal was observed in the 24 possible tests (three conditions $\times$ eight subjects). These data suggest that with a SNR cut off set high enough to exclude artefacts caused by noise, the occurrence of an interocular waveform polarity reversal is a fairly sensitive and specific indicator of a fixation error in the mfVEP.

\section{SUMMARY}

While the mfVEP is useful for identifying local optic nerve damage or ruling out non-organic aetiology of visual field defects, factors such as uncorrected refractive errors, cataract, and fixation errors can produce apparent field defects. With care, these problems can be correctly identified. When interpreting central abnormalities, it is important to rule out sources of blur such as incorrect refraction, voluntary or involuntary defocusing of the subject and lenticular opacity. If fixation artefacts are suspected, one should examine interocular waveforms for polarity reversals.

\section{ACKNOWLEDGEMENTS}

We thank Xian Zhang, Li Xu, and Annemarie Gallagher for their considerable help during all phases of this work.

\section{Authors' affiliations}

B J Winn, E Shin, D C Hood, Department of Psychology, Columbia University, New York, USA

J G Odel, V C Greenstein, Department of Ophthalmology, Columbia University, New York, USA

Supported by grant EY02115 from the National Institutes of Health, Bethesda, MD, USA.

\section{REFERENCES}

1 Sutter EE. The fast m-transform: a fast computation of cross-correlations with binary m-sequences. Soc Ind Appl Math 1991;20:686-94.

2 Baseler HA, Sutter EE, Klein SA, et al. The topography of visual evoked response properties across the visual field. Electroencephalogr Clin Neurophysiol 1994;90:65-81.

3 Graham SL, Klistorner Al, Grigg JR, et al. Objective VEP perimetry in glaucoma: asymmetry analysis to identify early deficits. J Glaucoma 2000;9:10-19.

4 Hood DC, Zhang X. Multifocal ERG and VEP responses and visual fields: comparing disease-related changes. Doc Ophthalmol 2000;100:115-37.

5 Hood DC, Zhang X, Greenstein VC, et al. An interocular comparison of the multifocal VEP: a possible technique for detecting local damage to the optic nerve. Invest Ophthalmol Vis Sci 2000;41:1580-7.
6 Goldberg I, Graham SL, Klistorner Al. Multifocal objective perimetry in the detection of glaucomatous field loss. Am J Ophthalmol 2002;133:29-39.

7 Hood DC, Zhang X, Winn BJ. Detecting glaucomatous damage with multifocal visual evoked potentials: how can a monocular test work? J Glaucoma 2003; 12:3-15.

8 Hood DC, Greenstein VC. Multifocal VEP and ganglion cell damage: applications and limitations for the study of glaucoma. Prog Retin Eye Res 2003;22:201-51.

9 Hood DC, Odel JG, Winn BJ. The multifocal visual evoked potential. J Neuroophthalmol 2003;23:279-89.

10 Hood DC, Odel JG, Zhang X. Tracking the recovery of local optic nerve function after optic neuritis: a multifocal VEP study. Invest Ophthalmol Vis Sci 2000;41:4032-8.

11 Miele DL, Odel JG, Behrens MM, et al. Functional bitemporal quadrantopia and the multifocal visual evoked potential. J Neuroophthalmol 2000;20:159-62.

12 Weinreb RN, Perlman JP. The effect of refractive correction on automated perimetric thresholds. Am J Ophthalmol 1986;101:706-9.

13 Wood JM, Wild JM, Smerdon DL, et al. Alterations in the shape of the automated perimetric profile arising from cataract. Graefes Arch Clin Exp Ophthalmol 1989;227:157-61.

14 Budenz DL, Feuer WJ, Anderson DR. The effect of simulated cataract on the glaucomatous visual field. Ophthalmology 1993;100:51 1-17.

15 Lam BL, Alward WL, Kolder HE. Effect of cataract on automated perimetry. Ophthalmology $1991 ; 98: 1066-70$.

16 Katz J, Sommer A. Reliability indexes of automated perimetric tests. Arch Ophthalmol 1988; 106:1252-4.

17 Sherman J. Visual evoked potential (VEP): basic concepts and clinical applications. J Am Optom Assoc 1979;50:19-30.

18 Hood DC, Zhang X, Hong JE, et al. Quantifying the benefits of additional channels of multifocal VEP recording. Doc Ophthalmol 2002; 104:303-20.

19 Zhang X, Hood DC, Chen CS, et al. A signal-to-noise analysis of multifocal VEP responses: an objective definition for poor records. Doc Ophthalmol 2002; 104:287-302

20 Chauhan BC, Henson DB, Hobley AJ. Cluster analysis in visual field quantification. Doc Ophthalmol 1988:69:25-39.

21 Katz J, Sommer A, Gaasterland DE, et al. Comparison of analytic algorithms for detecting glaucomatous visual field loss. Arch Ophthalmol $1991 ; 109: 1684-9$.

22 Fortune B, Goh K, Demirel S, et al. Detection of glaucomatous visual field loss using multifocal VEP. In: Wall M, Mills RP, eds. Perimetry Update 2002/3. The Hague: Kugler Publications (in press).

23 Hood DC, Thienprasiddhi P, Greenstein VC, et al. Detecting early to mild glaucomatous damage: a comparison of the multifocal VEP and automated perimetry. Invest Ophthalmol Vis Sci 2004;45:492-8.

24 Seiple W, Clemens C, Greenstein VC, et al. The spatial distribution of selective attention assessed using the multifocal visual evoked potential. Vis Res 2002;42:1513-21.

25 Menz MD, Poloschek CM, Menz MK, et al. The Effect Of Fixation Error On The Multifocal VEP. Association for Research in Vision and Ophthalmology 2002; abstract 4740

26 Slotnick SD, Klein SA, Carney T, et al. Using multi-stimulus VEP source localization to obtain a retinotopic map of human primary visual cortex. Clin Neurophysiol 1999;1 10:1793-800

27 Fortune B, Hood DC. Conventional pattern-reversal VEPs are not equivalent to summed multifocal VEPs. Invest Ophthalmol Vis Sci 2003;44:1364-75.

28 Zhang X, Hood DC. A principal component analysis of multifocal pattern reversal VEP. J Vis 2004;4:32-43.

29 Klistorner AI, Graham SL, Grigg JR, et al. Multifocal topographic visual evoked potential: improving objective detection of local visual field defects. Invest Ophthalmol Vis Sci 1998;39:937-50. 